\title{
Galactic Center: Implications of Recent Chandra Observations for Spherical Accretion Models of Sgr A*
}

\author{
Robert F. Coker \\ Department of Physics and Astronomy, University of Leeds, Leeds \\ LS2 9JT UK \\ Sera Markoff ${ }^{1}$ \\ Max-Planck-Institut für Radioastronomie, Auf dem Hügel 69, 53121 \\ Bonn, Germany
}

\begin{abstract}
At the center of the Milky Way lurks a unique compact nonthermal radio source, $\operatorname{Sgr} A^{*}$. It is thought to be powered by a $2.6 \times 10^{6}$ solar mass black hole that is accreting the stellar winds from the numerous early-type stars that exist in the central parsec. However, until recent high resolution Chandra observations, Sgr A* had never been unequivocably detected at wavelengths shorter than the sub-millimeter. We present a spherical accretion model which is consistent with both the flux and steep spectral shape of the X-ray emission from Sgr A*.
\end{abstract}

\section{The Spherical Accretion Model}

Sagittarius $A^{*}\left(\operatorname{Sgr} A^{*}\right)$, is located at the dynamical heart of the Milky Way (Reid et al. 1999; Backer \& Sramek 1999). Proper motion studies of stars in the Galactic Center (GC) indicate that $\mathrm{Sgr} \mathrm{A}^{*}$ is a $\sim 2.6 \times 10^{6} M_{\odot}$ black hole (e.g., Ghez et al. 2000; Genzel et al. 2000). There are more than two dozen known early-type stars in the central parsec (Genzel et al. 1996); combined, they produce winds that total $\sim 10^{-3} M_{\odot} \mathrm{yr}^{-1}$ (Najarro et al. 1997). In the classical Bondi-Hoyle scenario (Bondi \& Hoyle 1944), one would expect Sgr A* to be accreting a substantial fraction of these winds $\left(\sim 10^{-4} M_{\odot} \mathrm{yr}^{-1}\right)$. This picture is consistent with hydrodynamical simulations (Coker \& Melia 1997), although the precise accretion rate depends on the spatial distribution of the wind sources.

In our spherical accretion model (Coker \& Melia 2000), the radio spectrum of $\mathrm{Sgr} \mathrm{A}^{*}$ is due to magnetic bremsstrahlung. However, as the accreting winds fall down the gravitational well of $\mathrm{Sgr} \mathrm{A}^{*}$, the gas will also emit thermal bremsstrahlung, resulting in a significant X-ray flux. As pointed out by Quataert, Narayan, \& Reid (1999), X-ray observations can then be used to place limits on the accretion rate. In this work, we modify our model to satisfy the spectral index and flux limits of recent X-ray observations.

\footnotetext{
${ }^{1}$ Humboldt Research Fellow
} 
Initial ROSAT X-ray observations provided an upper limit on the luminosity of Sgr $\mathrm{A}^{*}$ of $\sim 4 L_{\odot}$ in the $0.8-2.5 \mathrm{keV}$ range (Predehl \& Zinnecker 1996). More recent, higher resolution Chandra observations show a source that is coincident with Sgr A* to within 1" (Baganoff et al. 2000). The luminosity of the source is $\sim 2 L_{\odot}$ in the $0.5-10 \mathrm{keV}$ range, suggesting that the ROSAT observations included either other sources or significant diffuse emission. Such a low luminosity leads to an upper limit on the accretion rate of $\sim 10^{-5} M_{\odot} \mathrm{yr}^{-1}$ (Quataert et al. 1999), significantly less than the predicted Bondi-Hoyle accretion rate. The X-ray spectrum of Sgr A* appears to be very soft, with a photon index of $\sim 2.75_{-1.00}^{+1.25}$ (Baganoff et al. 2000), while the spectrum of thermal bremsstrahlung emission is fairly flat until it cuts off exponentially at $h \nu \sim k T$. Since the radio emission requires relativistic temperatures, it is unlikely that the soft X-ray spectrum is due to thermal bremsstrahlung alone.

As discussed in Coker \& Melia (2000), the accretion flow that arises from nearby stars is assumed to be quasi-spherical, with no disk formation. We solve for the temperature and velocity profile, taking account of magnetic heating and radiative cooling. We find a sub-equipartition field at large radii with a nearly constant ratio of magnetic to thermal pressure, a constant field in a transition region, and a rapidly increasing field close to the event horizon. A dynamo at small radii is required to reproduce the "sub-mm bump" seen in the spectrum of Sgr A* (Serabyn et al. 1997; Falcke et al. 1998). Such a dynamo could easily be the result of instabilities in the flow which convert thermal energy to magnetic energy as the gas begins to circularize (Melia, Liu, \& Coker 2000).

This approach, however, is quite an oversimplification. In reality, even though the dynamo will transport angular momentum outwards (Balbus \& Hawley 1991), the flow is likely to have sufficient residual angular momentum to form a Keplerian flow at $r \lesssim 50 r_{s}$ where $r_{s} \equiv 2 G M / c^{2}$ is the Schwarzschild radius. However, unless the net accreted angular momentum vector is constant in time (see, e.g., Genzel et al. 2000), this flow is not likely to settle into a true accretion disk. We visualize an unhindered spherical flow at large radii, a smooth transition region where the flow circularizes, and an inner $\left(r \lesssim 3 r_{s}\right)$ region where the dynamo dominates; we thus model the magnetic field profile crudely as a series of three power laws.

As suggested by Falcke \& Markoff (2000), the soft X-ray spectrum is probably due to the up-scattering of sub-mm and infrared photons by synchrotronself-Compton (SSC). For a relativistic thermal distribution of particles, the frequency of the peak of the SSC spectrum will be $\sim 50\left(k T / m_{e} c^{2}\right)^{2} \nu_{s}$ where $T$ and $\nu_{s}$ are the characteristic temperature and frequency, respectively, of the magnetic bremsstrahlung emission. Fitting this to the observed spectrum suggests $T \sim 10^{11} \mathrm{~K}$, consistent with previous models (Coker \& Melia 2000) which did not, however, calculate the SSC spectrum explicitly.

\section{Results of SSC Model}

Following discussions during the conference, we have developed a spherical accretion model which, as a result of SSC, fits the flux and the spectral shape of the $\mathrm{X}$-ray observations while remaining consistent with the radio data. The input parameters are the magnetic field profile and the initial conditions at the outer 


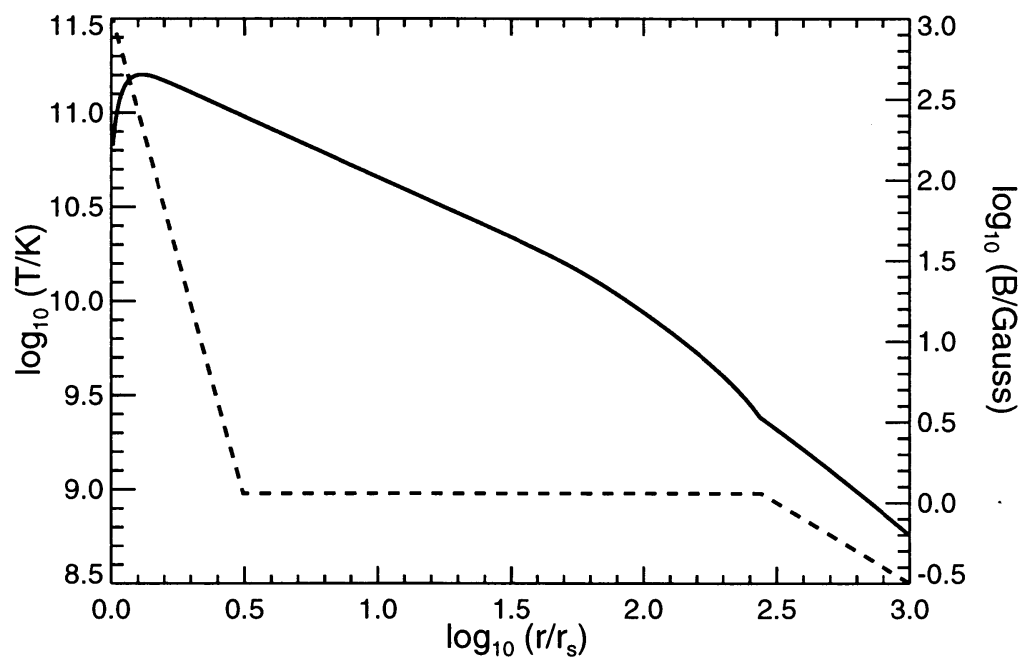

Figure 1. A plot of the temperature (solid line) and magnetic field (dashed line) versus radius for the best-fit model whose spectrum is shown in Figure 2. The central number density is $n_{\mathrm{H}} \simeq 6 \times 10^{8} \mathrm{~cm}^{-3}$ and corresponds to a mass accretion rate of $2 \times 10^{20} \mathrm{~g} \mathrm{~s}^{-1}$. For Sgr A*, $r_{s}=8 \times 10^{11} \mathrm{~cm}$.

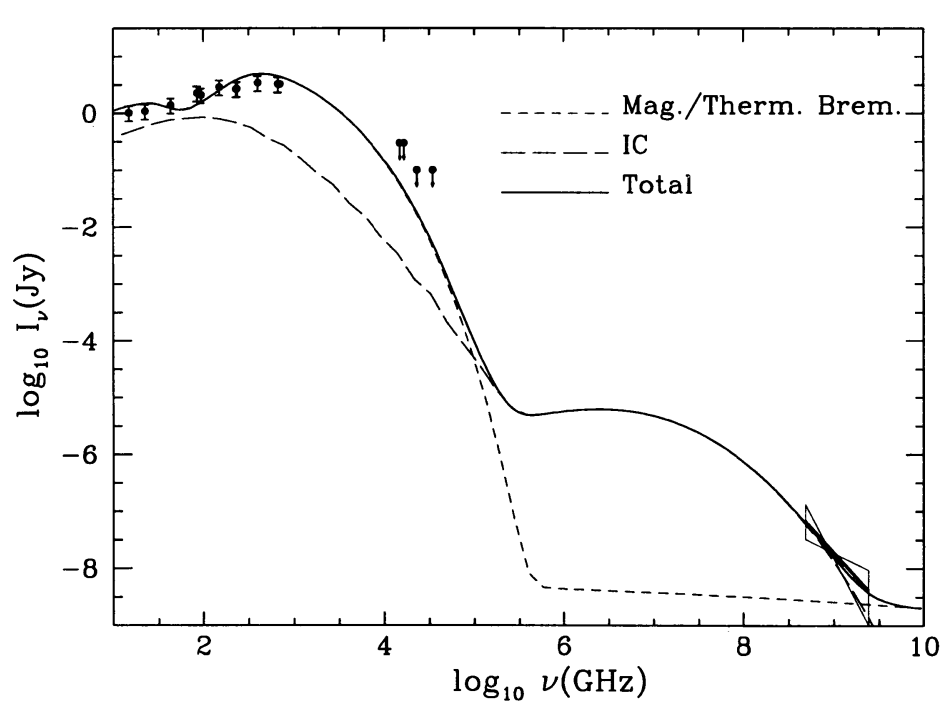

Figure 2. Best-fit combined thermal and magnetic bremsstrahlung, and Compton up-scattered, spectrum for Sgr A*. Data references in Falcke \& Markoff (2000). The error bars on the radio points correspond to $10 \%$ and are less than observed fluctuations (Zhao, Bower, \& Goss 2000). The detected Chandra power-law includes estimated errors in the spectral index (Baganoff et al. 2000). 
edge of the flow. The resulting radio and infrared emission spectra are then isotropically up-scattered via SSC by integrating over spherical shells, taking into account the self-absorption.

Our best-fit temperature and magnetic field profiles are presented in Figure 1 . The best-fit mass accretion rate is $3 \times 10^{-6} M_{\odot} \mathrm{yr}^{-1}$, a few times less than the upper limit set by Quataert et al. (1999), while the peak temperature and magnetic field are $3 \times 10^{11} \mathrm{~K}$ and $1000 \mathrm{G}$, respectively. Figure 2 shows the best-fit spectral components. The model satisfies the infrared upper limits, reproduces the X-ray spectral index and flux, and is consistent with the variable radio data.

It is not clear why the required accretion rate is so much lower than expected from HD simulations; perhaps there is an outflow, such as a small jet, resulting in a radially dependent accretion rate. Variability studies will be the key to discerning which model is correct. If there is no disk, variations in the flux from Sgr $\mathrm{A}^{*}$ will propagate from $\mathrm{cm}$ to $\mathrm{X}$-ray frequencies in less than a day. With a proper accretion disk, the lag between $\mathrm{cm}$ and X-ray fluctuations will be longer due to the reduced radial velocities. For a jet-like outflow, however, variations in the sub-mm will precede changes in the $\mathrm{cm}$ and X-ray.

\section{References}

Baganoff, F.K., et al. 2000, ApJ, submitted.

Balbus, S. A. \& Hawley, J. F. 1991, ApJ, 376, 214

Bondi, H. \& Hoyle, F. 1944, MNRAS, 104, 273

Coker, R.F. \& Melia, F. 1997, ApJ, 488, L149

Coker, R.F. \& Melia, F. 2000, ApJ, 534, 723

Falcke, H., et al. 1998, ApJ, 499, 731

Falcke, H. \& Markoff, S. 2000, A\&A, 362, 113

Genzel, R., et al. 1996, ApJ, 472, 153

Genzel, R., et al. 2000, MNRAS, 317, 348

Ghez, A.M., et al. 2000, Nature, 407, 349

Melia, F., Liu, S., \& Coker, R.F. 2000, ApJ, submitted.

Najarro, F., et al. 1997, A\&A, 325, 700

Quataert, E., Narayan, R., \& Reid, M.J. 1999, ApJ, 517, L101

Predehl, P. \& Zinnecker, H. 1996, in ASP Conf. Ser. Vol 102, The Galactic

Center, ed. R. Gredel (San Francisco: ASP), 415

Reid, M.J., et al. 1999, ApJ, 524, 816

Serabyn, E., et al. 1997, ApJ, 490, L77

Backer, D.C. \& Sramek, R.A. 1999, ApJ, 524, 805

Zhao, J.-H., Bower, GGG., \& Goss, W.M. 2000, ApJ, in press 